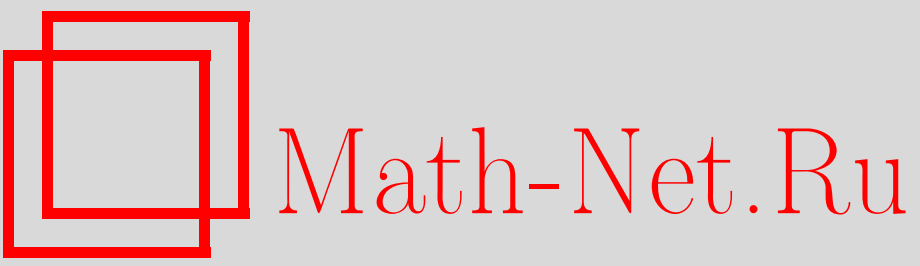

П. А. Вельмисов, А. В. Анкилов, Ю. В. Покладова, Об устойчивости решений некоторых классов начально-краевых задач в аэрогидроупругости, Итоги науки и техн. Сер. Соврем. мат. и ее прил. Темат. обз., 2019, том 165, 34-46

DOI: https://doi.org/10.36535/0233-6723-2019-165-34-46

Использование Общероссийского математического портала Math-Net.Ru подразумевает, что вы прочитали и согласны с пользовательским соглашением

http://www.mathnet.ru/rus/agreement

Параметры загрузки:

IP : 52.23 .180 .231

26 апреля 2023 г., 14:39:38 


\title{
ОБ УСТОЙЧИВОСТИ РЕШЕНИЙ НЕКОТОРЫХ КЛАССОВ НАЧАЛЬНО-КРАЕВЫХ ЗАДАЧ В АЭРОГИДРОУПРУГОСТИ
}

\author{
(c) 2019 г. П. А. ВЕЛЬМИСОВ, А. В. АНКИЛОВ, Ю. В. ПОКЛАДОВА
}

\begin{abstract}
АннотАция. Исследуется устойчивость решений начально-краевых задач для связанных систем дифференциальных уравнений с частными производными, описывающих динамику деформируемых элементов конструкций, находящихся во взаимодействии с газожидкостной средой (обтекаемых потоком жидкости или газа). Принятые в работе определения устойчивости деформируемого тела соответствуют концепции устойчивости динамических систем по Ляпунову. Исследована устойчивость деформируемых элементов вибрационного устройства, взаимодействующих с дозвуковым потоком, и деформируемого элемента конструкции при обтекании его сверхзвуковым потоком. Воздействие газа или жидкости (в модели идеальной сжимаемой среды) определяется из асимптотических уравнений аэрогидромеханики. Для описания динамики упругих элементов использованы нелинейные модели твердого деформируемого тела, учитывающие их поперечные и продольные деформации. Модели описываются связанными нелинейными системами дифференциальных уравнений в частных производных. Исследование устойчивости проводится на основе построения положительно определенных функционалов типа Ляпунова, соответствующих этим системам, получены достаточные условия устойчивости их решений.
\end{abstract}

Ключевъе слова: аэрогидроупругость, математическое моделирование, динамическая устойчивость, упругая пластина, дозвуковой поток, сверхзвуковой поток, дифференциальное уравнение в частных производных, функционал.

\section{ON THE STABILITY OF SOLUTIONS OF CERTAIN CLASSES OF INITIAL-BOUNDARY-VALUE PROBLEMS IN AEROHYDROELASTICITY}

\author{
(c) 2019 P. A. VELMISOV, A. V. ANKILOV, YU. V. POKLADOVA
}

\begin{abstract}
We study the stability of solutions to initial-boundary-value problems for coupled systems of partial differential equations describing the dynamics of deformable structural elements interacting with a gas-liquid medium. The definitions of stability of deformable bodied adopted in this work correspond to the concept of the Lyapunov stability of dynamic systems. The stability of deformable elements of vibration devices interacting with subsonic and supersonic flows is examined. The effect of a gas or liquid (in the model of an ideal compressible medium) is determined from asymptotic equations of aerohydromechanics. For the description of the dynamics of elastic elements, we use nonlinear models of solid deformable bodies with transverse and longitudinal deformations. Models are described by coupled nonlinear systems of partial differential equations. The study of stability is based on the construction of positive-definite Lyapunov-type functionals corresponding to these systems; sufficient conditions for the stability of their solutions are obtained.
\end{abstract}

Keywords and phrases: aerohydroelasticity, mathematical modeling, dynamic stability, elastic plate, subsonic flow, supersonic flow, partial differential equation, functional.

AMS Subject Classification: $74 \mathrm{~F} 10$

Работа выполнена при поддержке Российского фонда фундаментальных исследований (проект № 18-41-730015). 
1. Введение. При проектировании и эксплуатации конструкций, приборов, устройств, установок различного назначения, взаимодействующих с потоком газа или жидкости, важной проблемой является обеспечение надежности их функционирования и увеличение сроков службы. Подобные проблемы присущи многим отраслям техники. В частности, такого рода задачи возникают в авиаракетостроении, приборостроении, при проектировании антенных установок, высоких наземных сооружений и т. д.

В настоящее время аэрогидроупругость представляет собой хорошо развитый раздел механики сплошной среды. Много исследований посвящено динамике, устойчивости и флаттеру пластин и оболочек, находящихся в потоке жидкости или газа (среди последних в качестве примера отметим как российские $[1,9,10,12,13,22,26]$, так и зарубежные $[15-21,23-25,27]$ исследования). В связи с развитием компьютерных технологий в подавляющем большинстве последних работ для исследования динамики и устойчивости используются численные методы расчета. Однако много работ посвящено и аналитическому исследованию линейных моделей аэрогидроупругости с применением частотных методов. В данной статье для описания динамики упругого элемента предлагается нелинейная модель, учитывающая как поперечные, так и продольные колебания пластины. Это делает невозможным использование частотных методов и усложняет численное решение поставленной задачи, так как приводит к продолжительным расчетам и большим погрешностям в этих расчетах.

В работе исследуется устойчивость решений начально-краевых задач для связанных систем дифференциальных уравнений с частными производными, описывающих динамику деформируемых элементов конструкций, находящихся во взаимодействии с газожидкостной средой (обтекаемых потоком жидкости или газа). Исследование устойчивости проводится на основе построения положительно определенных функционалов (прямой метод Ляпунова) для этих систем. Принятые в работе определения устойчивости деформируемого тела соответствуют концепции устойчивости динамических систем по Ляпунову. Проблема может быть сформулирована так: при каких значениях параметров, характеризующих систему «жидкость-тело» (основными параметрами являются скорость потока, прочностные и инерционные характеристики тела, сжимающие или растягивающие усилия, силы трения), малым деформациям тел в начальный момент времени $t=0$ (т.е. малым начальным отклонениям от положения равновесия) будут соответствовать малые деформации и в любой момент времени $t>0$. Среди работ авторов данной статьи по исследованию динамики и устойчивости упругих тел, взаимодействующих с потоком жидкости или газа, отметим монографии и статьи $[2-8,14]$.

Изучается устойчивость элементов конструкций при жестком закреплении их концов при дозвуковом или сверхзвуковом режимах обтекания газожидкостной средой. Воздействие газа или жидкости (в модели идеальной среды) определяется из асимптотических уравнений аэрогидромеханики. В качестве примера дозвукового обтекания приведены результаты исследования динамической устойчивости деформируемых элементов вибрационных устройств. Основной частью широкого класса этих устройств является проточный канал, внутри которого расположены деформируемые элементы. Работа таких устройств основана на вибрации деформируемых элементов при протекании внутри каналов жидкости. В качестве примера сверхзвукового обтекания приведены результаты исследования динамической устойчивости деформируемого упругого защитного экрана.

\section{2. Динамическая устойчивость деформируемых элементов вибрационного устрой- ства при взаимодействии с дозвуковым потоком.}

Математическая модель. Рассмотрим плоское течение в вибрационном устройстве, моделируемом прямолинейным каналом $G=\left\{(x, y) \in \mathbb{R}^{2}: 0<x<x_{0}, 0<y<y_{0}\right\}$ с горизонтальными недеформируемыми стенками $y=0$ и $y=y_{0}$. Внутри канала расположены два параллельных деформируемых упругих элемента. Скорость невозмущенного однородного потока равна $V$ и направлена вдоль оси $O x$. Рассматривается дозвуковой режим протекания $a>V$, где $a$-скорость звука в невозмущенном потоке жидкости. Деформируемыми являются пластины, занимающие положение $y=y_{1}$ и $y=y_{2}, x \in[b, c]$ (см. рис. 1 ). Введем обозначение 


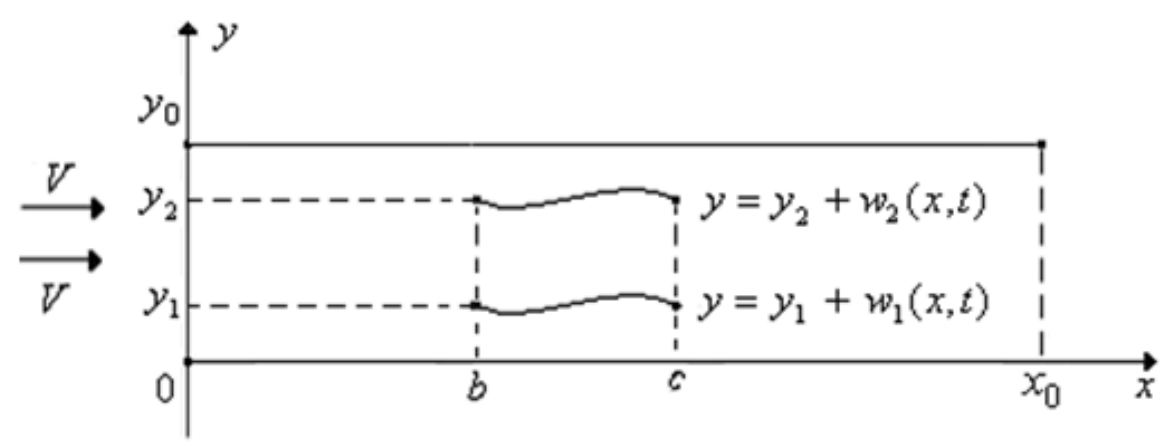

Рис. 1. Канал, внутри которого расположены параллельно деформируемые элементы.

$$
J_{i}=\left\{(x, y) \in \mathbb{R}^{2}: y=y_{i} \in\left(0, y_{0}\right), x \in[b, c]\right\}, \quad i=1,2, \quad J=J_{1} \cup J_{2} .
$$

Введем также деформации $u_{i}(x, t)$ и $w_{i}(x, t), i=1,2$, упругих элементов в направлении осей $O x$ и $O y$ соответственно (индекс $i=1$ соответствует элементу при $y=y_{1}, i=2-$ при $y=y_{2}$ ) и потенциал скорости возмущенного потока $\varphi(x, y, t)$.

Сформулируем математическую постановку задачи:

$$
\begin{gathered}
\varphi_{t t}+2 V \varphi_{x t}+V^{2} \varphi_{x x}=a^{2}\left(\varphi_{x x}+\varphi_{y y}\right), \quad(x, y) \in G \backslash J, \quad t \geqslant 0, \\
\varphi_{y}\left(x, y_{0}, t\right)=0, \quad x \in\left(0, x_{0}\right), \quad t \geqslant 0, \\
\varphi_{y}(x, 0, t)=0, \quad x \in\left(0, x_{0}\right), \quad t \geqslant 0, \\
\dot{w}_{y}\left(x, y_{i}, t\right)=\dot{w}_{i}(x, t)+V w_{i}^{\prime}(x, t), \quad i=1,2, \quad x \in(b, c), \quad t \geqslant 0, \\
\varphi(0, y, t)=0, \quad \varphi\left(x_{0}, y, t\right)=0, \quad y \in\left(0, y_{0}\right), \\
P_{i}(x, t)=\rho\left(\varphi_{t}^{+}\left(x, y_{i}, t\right)-\varphi_{t}^{-}\left(x, y_{i}, t\right)\right)+\rho V\left(\varphi_{x}^{+}\left(x, y_{i}, t\right)-\varphi_{x}^{-}\left(x, y_{i}, t\right)\right), \quad x \in(b, c), \quad t \geqslant 0,
\end{gathered}
$$

Индексы $x, y, t$ снизу обозначают частные производные по $x, y, t$; штрих и точка-частные производные по $x$ и $t$ соответственно; $\rho$ - плотность потока; $a$-скорость звука в невозмущенном потоке газожидкостной среды $(a>V)$;

$$
\varphi_{t}^{ \pm}\left(x, y_{i}, t\right)=\lim _{y \rightarrow y_{i} \pm 0} \varphi_{t}(x, y, t), \quad \varphi_{x}^{ \pm}\left(x, y_{i}, t\right)=\lim _{y \rightarrow y_{i} \pm 0} \varphi_{x}(x, y, t) .
$$

Рассмотрим нелинейную модель колебаний упругого тела с учетом продольных и поперечных составляющих деформаций элементов, моделируемых упругими пластинами, с учетом силового воздействия потока $P_{i}(x, t)$ на них. Тогда математическую постановку задачи $(2.1)-(2.6)$ следует дополнить уравнениями

$$
\left\{\begin{array}{l}
-E_{i} F_{i}\left(u_{i}^{\prime}(x, t)+\frac{1}{2} w_{i}^{\prime 2}(x, t)\right)^{\prime}+M_{i} \ddot{u}_{i}(x, t)=0, \\
-E_{i} F_{i}\left[w_{i}^{\prime}(x, t)\left(u_{i}^{\prime}(x, t)+\frac{1}{2} w_{i}^{\prime 2}(x, t)\right)\right]^{\prime}+D_{i} w_{i}^{\prime \prime \prime \prime}(x, t)+M_{i} \ddot{w}_{i}(x, t)+N_{i}(t) w_{i}^{\prime \prime}(x, t)+ \\
\quad+\beta_{2 i} \dot{w}_{i}^{\prime \prime \prime \prime}(x, t)+\beta_{1 i} \dot{w}_{i}(x, t)+\beta_{0 i} w_{i}(x, t)=P_{i}(x, t), \quad x \in(b, c), \quad i=1,2, \quad t \geqslant 0 .
\end{array}\right.
$$

Здесь $D_{i}=E_{i}{ }^{3} /\left(12\left(1-\nu_{i}^{2}\right)\right)$ - изгибные жесткости элементов; $h_{i}$-толщина элементов; $M_{i}=$ $h_{i} \rho_{i}$ - погонные массы элементов; $E_{i}, \rho_{i}$ - модули упругости и линейные плотности элементов; $F_{i}=h_{i} /\left(1-\nu_{i}^{2}\right) ; N_{i}(t)$ - сжимающие $\left(N_{i}>0\right)$ или растягивающие $\left(N_{i}<0\right)$ элементы силы; $\beta_{2 i}$, $\beta_{1 i}$ - коэффициенты внутреннего и внешнего демпфирования; $\beta_{0 i}$-коэффициенты жесткости слоя обжатия; $\nu_{i}$ - коэффициенты Пуассона. 
Сжимающие или растягивающие пластины силы $N_{i}$ могут зависеть от времени. Например, при изменении теплового воздействия на пластины с течением времени $N_{i}(t)$ имеют вид

$$
N_{i}(t)=N_{0 i}+N_{T i}(t), \quad N_{T i}(t)=\frac{T_{0 i}(t)}{1-\nu_{i}}, \quad T_{0 i}(t)=E_{i} \alpha_{T i} \int_{-h_{i} / 2}^{h_{i} / 2} T_{i}(z, t) d z,
$$

где $\alpha_{T i}$ - температурные коэффициенты линейного расширения, $T_{i}(z, t)$ - законы изменения температуры по толщине пластин, $N_{0 i}$ - постоянные составляющие усилий, созданные при закреплении пластин.

Исследуем динамическую устойчивость упругих элементов в случае жесткого защемления их концов:

$$
w_{i}(b, t)=w_{i}^{\prime}(b, t)=u_{i}(b, t)=w_{i}(c, t)=w_{i}^{\prime}(c, t)=u_{i}(c, t)=0, \quad i=1,2 .
$$

Имеем связанную краевую задачу (2.1)-(2.8) для пяти неизвестных функций - деформаций упругих элементов $u_{i}(x, t), w_{i}(x, t), i=1,2$, и потенциала скорости жидкости (газа) $\varphi(x, y, t)$.

Зададим начальные условия:

$$
\begin{gathered}
w_{i}(x, 0)=f_{1 i}(x), \quad \dot{w}_{i}(x, 0)=f_{2 i}(x), \quad u_{i}(x, 0)=f_{3 i}(x), \quad \dot{u}_{i}(x, 0)=f_{4 i}(x), \quad i=1,2, \\
\varphi(x, y, 0)=f_{5}(x, y), \quad \varphi_{t}(x, y, 0)=f_{6}(x, y),
\end{gathered}
$$

которые должны быть согласованы с краевыми условиями.

Исследование устойчивости. Так как задача (2.1)-(2.8) однородная, исследуем устойчивость ее нулевого решения $\varphi(x, y, t) \equiv 0, w_{i}(x, t) \equiv 0, u_{i}(x, t) \equiv 0, i=1,2$, по отношению к возмущениям начальных данных. Рассмотрим функционал

$$
\begin{aligned}
& \Phi(t)=\iint_{G \backslash J}\left(\varphi_{t}^{2}(x, y, t)\right.\left.+\left(a^{2}-V^{2}\right) \varphi_{x}^{2}(x, y, t)+a^{2} \varphi_{y}^{2}(x, y, t)\right) d x d y+ \\
&+2 a^{2} V \sum_{i=1}^{2} \int_{b}^{c}\left(\varphi^{+}\left(x, y_{i}, t\right)-\varphi^{-}\left(x, y_{i}, t\right)\right) w_{i}^{\prime}(x, t) d x+ \\
&+\frac{a^{2}}{\rho} \sum_{i=1}^{2} \int_{b}^{c}\left(E_{i} F_{i}\left(u_{i}^{\prime}(x, t)+\frac{1}{2} w_{i}^{\prime 2}(x, t)\right)^{2}+M\left(\dot{u}_{i}^{2}(x, t)+\dot{w}_{i}^{2}(x, t)\right)+D_{i} w_{i}^{\prime \prime 2}(x, t)+\right. \\
&\left.+\beta_{0 i} w_{i}^{2}(x, t)-N_{i}(t) w_{i}^{\prime 2}(x, t)\right) d x .
\end{aligned}
$$

Найдем производную от $\Phi$ по $t$ :

$$
\begin{aligned}
& \dot{\Phi}(t)=2 \iint_{G \backslash J}\left(\varphi_{t} \varphi_{t t}+\left(a^{2}-V^{2}\right) \varphi_{x} \varphi_{x t}+a^{2} \varphi_{y} \varphi_{y t}\right) d x d y+ \\
&+2 a^{2} V \sum_{i=1}^{2} \int_{b}^{c}\left(\left(\varphi_{t}^{+}\left(x, y_{i}, t\right)-\varphi_{t}^{-}\left(x, y_{i}, t\right)\right) w_{i}^{\prime}(x, t)+\left(\varphi^{+}\left(x, y_{i}, t\right)-\varphi^{-}\left(x, y_{i}, t\right)\right) \dot{w}_{i}^{\prime}(x, t)\right) d x+ \\
&+\frac{2 a^{2}}{\rho} \sum_{i=1}^{2} \int_{b}^{c}\left(E_{i} F_{i}\left(u_{i}^{\prime}+\frac{1}{2}{w_{i}^{\prime}}^{2}\right)\left(\dot{u}_{i}^{\prime}+w_{i}^{\prime} \dot{w}_{i}^{\prime}\right)+M \dot{u}_{i} \ddot{u}_{i}+M_{i} \dot{w}_{i} \ddot{w}_{i}+D_{i} w_{i}^{\prime \prime} \dot{w}_{i}^{\prime \prime}-\right. \\
&\left.-\frac{1}{2} \dot{N}_{i}(t) w_{i}^{\prime 2}-N_{i}(t) w_{i}^{\prime} \dot{w}_{i}^{\prime}+\beta_{0 i} w_{i} \dot{w}_{i}\right) d x
\end{aligned}
$$


Для функций $\varphi(x, y, t)$ и $w_{i}(x, t), u_{i}(x, t), i=1,2$, удовлетворяющих уравнениям $(2.1),(2.6),(2.7)$, равенство (2.12) примет вид

$$
\dot{\Phi}(t)=-\frac{2 a^{2}}{\rho} \sum_{i=1}^{2} \int_{b}^{c}\left(\frac{1}{2} \dot{N}_{i}(t) w_{i}^{\prime 2}+\beta_{2 i}\left(\dot{w}_{i}^{\prime \prime}\right)^{2}+\beta_{1 i}\left(\dot{w}_{i}\right)^{2}\right) d x .
$$

Пусть выполняются условия

$$
\dot{N}_{i}(t) \geqslant 0, \quad \beta_{2 i} \geqslant 0, \quad \beta_{1 i} \geqslant 0, \quad i=1,2 ;
$$

тогда

$$
\dot{\Phi}(t) \leqslant 0
$$

Проведем оценки для функционала с учетом граничных условий (2.8). Воспользуемся неравенством Рэлея:

$$
\int_{b}^{c}\left(w_{i}^{\prime \prime}\right)^{2}(x, t) d x \geqslant \lambda_{1 i} \int_{b}^{c}\left(w_{i}^{\prime}\right)^{2}(x, t) d x, \quad i=1,2,
$$

где $\lambda_{1 i}$ - наименьшие собственные значения (см. [11]) краевых задач для уравнения $\psi^{\prime \prime \prime \prime}=-\lambda \psi^{\prime \prime}$, $x \in(b, c)$ с краевыми условиями $\psi(b)=\psi^{\prime}(b)=\psi(c)=\psi^{\prime}(c)=0$, соответствующими условиям $(2.8)$ для функций $w_{i}(x, t)$.

Оценим $\Phi(t)$ снизу:

$$
\begin{aligned}
\Phi(t) \geqslant \iint_{G \backslash J}\left(\varphi_{t}^{2}+\left(a^{2}-V^{2}\right) \varphi_{x}^{2}+a^{2} \varphi_{y}^{2}\right) d x d y+ & \\
& +2 a^{2} V \sum_{i=1}^{2} \int_{b}^{c}\left(\varphi^{+}\left(x, y_{i}, t\right)-\varphi^{-}\left(x, y_{i}, t\right)\right) w_{i}^{\prime}(x, t) d x+ \\
& +\frac{a^{2}}{\rho} \sum_{i=1}^{2} \int_{b}^{c}\left(\lambda_{1 i} D_{i}-N_{i}(t)\right)\left(w_{i}^{\prime}\right)^{2} d x .
\end{aligned}
$$

Для оценки двойного интеграла разобьем область $G \backslash J$ на три области

$$
\begin{aligned}
& G_{1}=\left\{(x, y) \in \mathbb{R}^{2}: 0<x<x_{0}, 0<y<y_{1}\right\}, \\
& G_{2}=\left\{(x, y) \in \mathbb{R}^{2}: 0<x<x_{0}, y_{1}<y<y_{2}\right\}, \\
& G_{3}=\left\{(x, y) \in \mathbb{R}^{2}: 0<x<x_{0}, y_{2}<y<y_{0}\right\} .
\end{aligned}
$$

Согласно неравенству Коши-Буняковского

$$
\begin{gathered}
\iint_{G_{1}} \varphi_{x}^{2} d x d y \geqslant \frac{\pi^{2}}{x_{0}^{2}} \iint_{G_{1}} \varphi^{2} d x d y, \iint_{G_{2}} \varphi_{x}^{2} d x d y \geqslant \frac{\pi^{2}}{x_{0}^{2}} \iint_{G_{2}} \varphi^{2} d x d y, \iint_{G_{3}} \varphi_{x}^{2} d x d y \geqslant \frac{\pi^{2}}{x_{0}^{2}} \iint_{G_{3}} \varphi^{2} d x d y \\
\iint_{G_{1}} \varphi_{y}^{2} d x d y \geqslant \frac{2}{y_{1}^{2}} \iint_{G_{1}}\left(\varphi^{-}\left(x, y_{1}, t\right)-\varphi(x, y, t)\right)^{2} d x d y \\
\iint_{G_{2}} \varphi_{y}^{2} d x d y \geqslant \frac{2 \chi_{1}(t)}{\left(y_{2}-y_{1}\right)^{2}} \iint_{G_{2}}\left(\varphi(x, y, t)-\varphi^{+}\left(x, y_{1}, t\right)\right)^{2} d x d y+ \\
+\frac{2\left(1-\chi_{1}(t)\right)}{\left(y_{2}-y_{1}\right)^{2}} \iint_{G_{2}}\left(\varphi^{-}\left(x, y_{2}, t\right)-\varphi(x, y, t)\right)^{2} d x d y
\end{gathered}
$$




$$
\iint_{G_{3}} \varphi_{y}^{2} d x d y \geqslant \frac{2}{\left(y_{0}-y_{2}\right)^{2}} \iint_{G_{3}}\left(\varphi(x, y, t)-\varphi^{+}\left(x, y_{2}, t\right)\right)^{2} d x d y
$$

где $\chi_{1}(t) \in(0,1)$ - произвольная функция переменной $t$.

Введем обозначения

$$
K_{i}(t)=\lambda_{1 i} D_{i}-N_{i}(t), \quad f_{i}(x, t)=\left\{\begin{array}{l}
0, \quad x \in(0, b] \cup\left[c, x_{0}\right) \\
w_{i}^{\prime}(x, t), \quad x \in(b, c)
\end{array}\right.
$$

Тогда, применяя (2.17)-(2.20) к (2.16), получим следующее неравенство:

$$
\begin{aligned}
& \Phi(t) \geqslant \iint_{G_{1}}\left(\varphi_{t}^{2}+\left(a^{2}-V^{2}\right) \frac{\pi^{2}}{x_{0}^{2}} \varphi^{2}+\frac{2 a^{2}}{y_{1}^{2}}\left(\varphi^{-}\left(x, y_{1}, t\right)-\varphi(x, y, t)\right)^{2}-\right. \\
& \left.-\frac{2 a^{2} V}{y_{1}} \varphi^{-}\left(x, y_{1}, t\right) f_{1}(x, t)+\frac{a^{2} \chi_{2}(t)}{\rho y_{1}} K_{1}(t) f_{1}^{2}(x, t)\right) d x d y+ \\
& \quad+\chi_{1}(t) \iint_{G_{2}}\left(\varphi_{t}^{2}+\left(a^{2}-V^{2}\right) \frac{\pi^{2}}{x_{0}^{2}} \varphi^{2}+\frac{2 a^{2}}{\left(y_{2}-y_{1}\right)^{2}}\left(\varphi(x, y, t)-\varphi^{+}\left(x, y_{1}, t\right)\right)^{2}+\right. \\
& \left.+\frac{2 a^{2} V}{\chi_{1}(t)\left(y_{2}-y_{1}\right)} \varphi^{+}\left(x, y_{1}, t\right) f_{1}(x, t)+\frac{a^{2}\left(1-\chi_{2}(t)\right)}{\rho\left(y_{2}-y_{1}\right) \chi_{1}(t)} K_{1}(t) f_{1}^{2}(x, t)\right) d x d y+ \\
& \quad+\left(1-\chi_{1}(t)\right) \iint_{G_{2}}\left(\varphi_{t}^{2}+\left(a^{2}-V^{2}\right) \frac{\pi^{2}}{x_{0}^{2}} \varphi^{2}+\frac{2 a^{2}}{\left(y_{2}-y_{1}\right)^{2}}\left(\varphi^{-}\left(x, y_{2}, t\right)-\varphi(x, y, t)\right)^{2}-\right. \\
& \left.-\frac{2 a^{2} V}{\left(1-\chi_{1}(t)\right)\left(y_{2}-y_{1}\right)} \varphi^{-}\left(x, y_{2}, t\right) f_{2}(x, t)+\frac{a^{2} \chi_{3}(t)}{\rho\left(y_{2}-y_{1}\right)\left(1-\chi_{1}(t)\right)} K_{2}(t) f_{2}^{2}(x, t)\right) d x d y+ \\
& \quad+\iint_{G_{3}}\left(\varphi_{t}^{2}+\left(a^{2}-V^{2}\right) \frac{\pi^{2}}{x_{0}^{2}} \varphi^{2}+\frac{2 a^{2}}{\left(y_{0}-y_{2}\right)^{2}}\left(\varphi(x, y, t)-\varphi^{+}\left(x, y_{2}, t\right)\right)^{2}+\right. \\
& \left.\quad+\frac{2 a^{2} V}{y_{0}-y_{2}} \varphi^{+}\left(x, y_{2}, t\right) f_{2}(x, t)+\frac{a^{2}\left(1-\chi_{3}(t)\right)}{\rho\left(y_{0}-y_{2}\right)} K_{2}(t) f_{2}^{2}(x, t)\right) d x d y,
\end{aligned}
$$

где $\chi_{2}(t) \in(0,1), \chi_{3}(t) \in(0,1)$ - произвольные функции переменной $t$.

Рассмотрим квадратичные формы относительно $\varphi(x, y, t), \varphi^{-}\left(x, y_{1}, t\right), f_{1}(x, t)$ и $\varphi(x, y, t)$, $\varphi^{+}\left(x, y_{1}, t\right), f_{1}(x, t)$ в $(2.21)$. Пользуясь критерием Сильвестра, запишем условия положительной определенности квадратичных форм:

$$
\begin{gathered}
K_{1}(t)>\frac{V^{2} x_{0}^{2} \rho y_{1}}{2\left(a^{2}-V^{2}\right) \pi^{2} \chi_{2}(t)}\left(\frac{\left(a^{2}-V^{2}\right) \pi^{2}}{x_{0}^{2}}+\frac{2 a^{2}}{y_{1}^{2}}\right), \\
K_{1}(t)>\frac{V^{2} x_{0}^{2} \rho\left(y_{2}-y_{1}\right)}{2\left(a^{2}-V^{2}\right) \pi^{2}\left(1-\chi_{2}(t)\right) \chi_{1}(t)}\left(\frac{\left(a^{2}-V^{2}\right) \pi^{2}}{x_{0}^{2}}+\frac{2 a^{2}}{\left(y_{2}-y_{1}\right)^{2}}\right) .
\end{gathered}
$$

Из неравенств $(2.22),(2.23)$ найдем оптимальную функцию $\chi_{2}(t)$, обеспечивающую наиболее широкую область значений параметров, входящих в условия (2.22), (2.23). Для этого приравняем их правые части:

$$
\begin{aligned}
\frac{V^{2} x_{0}^{2} \rho y_{1}}{2\left(a^{2}-V^{2}\right) \pi^{2} \chi_{2}(t)}\left(\frac{\left(a^{2}-V^{2}\right) \pi^{2}}{x_{0}^{2}}\right. & \left.+\frac{2 a^{2}}{y_{1}^{2}}\right)= \\
& =\frac{V^{2} x_{0}^{2} \rho\left(y_{2}-y_{1}\right)}{2\left(a^{2}-V^{2}\right) \pi^{2}\left(1-\chi_{2}(t)\right) \chi_{1}(t)}\left(\frac{\left(a^{2}-V^{2}\right) \pi^{2}}{x_{0}^{2}}+\frac{2 a^{2}}{\left(y_{2}-y_{1}\right)^{2}}\right) .
\end{aligned}
$$

Тогда получим

$$
\chi_{2}(t)=\frac{\chi_{1}(t)\left(y_{2}-y_{1}\right)\left(\left(a^{2}-V^{2}\right) \pi^{2} y_{1}^{2}+2 a^{2} x_{0}^{2}\right)}{y_{1}\left(\left(a^{2}-V^{2}\right) \pi^{2}\left(y_{2}-y_{1}\right)^{2}+2 a^{2} x_{0}^{2}\right)+\chi_{1}(t)\left(y_{2}-y_{1}\right)\left(\left(a^{2}-V^{2}\right) \pi^{2} y_{1}^{2}+2 a^{2} x_{0}^{2}\right)} .
$$


Подставляя (2.24) в (2.22) и (2.23), приходим к выводу, что оба эти условия примут вид

$$
K_{1}(t)>\frac{V^{2} \rho\left(\left(a^{2}-V^{2}\right) \pi^{2}\left(y_{2}-y_{1}\right)^{2}+2 a^{2} x_{0}^{2}\right)}{2 \chi_{1}(t)\left(a^{2}-V^{2}\right) \pi^{2}\left(y_{2}-y_{1}\right)}+\frac{V^{2} \rho\left(\left(a^{2}-V^{2}\right) \pi^{2} y_{1}^{2}+2 a^{2} x_{0}^{2}\right)}{2\left(a^{2}-V^{2}\right) \pi^{2} y_{1}} .
$$

Рассмотрим квадратичные формы относительно $\varphi(x, y, t), \varphi^{-}\left(x, y_{2}, t\right), f_{2}(x, t)$ и $\varphi(x, y, t)$, $\varphi^{+}\left(x, y_{2}, t\right), f_{2}(x, t)$ в $(2.21)$. Пользуясь критерием Сильвестра, запишем условия положительной определенности квадратичных форм:

$$
\begin{gathered}
K_{2}(t)>\frac{V^{2} x_{0}^{2} \rho\left(y_{2}-y_{1}\right)}{2\left(a^{2}-V^{2}\right) \pi^{2}\left(1-\chi_{1}(t)\right) \chi_{3}(t)}\left(\frac{\left(a^{2}-V^{2}\right) \pi^{2}}{x_{0}^{2}}+\frac{2 a^{2}}{\left(y_{2}-y_{1}\right)^{2}}\right), \\
K_{2}(t)>\frac{V^{2} x_{0}^{2} \rho\left(y_{0}-y_{2}\right)}{2\left(a^{2}-V^{2}\right) \pi^{2}\left(1-\chi_{3}(t)\right)}\left(\frac{\left(a^{2}-V^{2}\right) \pi^{2}}{x_{0}^{2}}+\frac{2 a^{2}}{\left(y_{0}-y_{2}\right)^{2}}\right) .
\end{gathered}
$$

Из неравенств $(2.26),(2.27)$ найдем оптимальную функцию $\chi_{3}(t)$, обеспечивающую наиболее широкую область значений параметров, входящих в условия (2.26), (2.27). Для этого приравняем их правые части:

$$
\begin{aligned}
\frac{V^{2} x_{0}^{2} \rho\left(y_{2}-y_{1}\right)}{2\left(a^{2}-V^{2}\right) \pi^{2}\left(1-\chi_{1}(t)\right) \chi_{3}(t)}\left(\frac{\left(a^{2}-V^{2}\right) \pi^{2}}{x_{0}^{2}}+\frac{2 a^{2}}{\left(y_{2}-y_{1}\right)^{2}}\right)= \\
\quad=\frac{V^{2} x_{0}^{2} \rho\left(y_{0}-y_{2}\right)}{2\left(a^{2}-V^{2}\right) \pi^{2}\left(1-\chi_{3}(t)\right)}\left(\frac{\left(a^{2}-V^{2}\right) \pi^{2}}{x_{0}^{2}}+\frac{2 a^{2}}{\left(y_{0}-y_{2}\right)^{2}}\right) .
\end{aligned}
$$

Тогда получим

$$
\begin{aligned}
\chi_{3}(t)= & \left(\left(y_{0}-y_{2}\right)\left(\left(a^{2}-V^{2}\right) \pi^{2}\left(y_{2}-y_{1}\right)^{2}+2 a^{2} x_{0}^{2}\right)\right) /\left(\left(1-\chi_{1}(t)\right)\left(y_{2}-y_{1}\right) \times\right. \\
& \left.\times\left(\left(a^{2}-V^{2}\right) \pi^{2}\left(y_{0}-y_{2}\right)^{2}+2 a^{2} x_{0}^{2}\right)+\left(y_{0}-y_{2}\right)\left(\left(a^{2}-V^{2}\right) \pi^{2}\left(y_{2}-y_{1}\right)^{2}+2 a^{2} x_{0}^{2}\right)\right) .
\end{aligned}
$$

Подставляя (2.28) в (2.26) и (2.27), приходим к выводу, что оба эти условия примут вид

$$
K_{2}(t)>\frac{V^{2} \rho\left(\left(a^{2}-V^{2}\right) \pi^{2}\left(y_{0}-y_{2}\right)^{2}+2 a^{2} x_{0}^{2}\right)}{2\left(y_{0}-y_{2}\right)\left(a^{2}-V^{2}\right) \pi^{2}}+\frac{V^{2} \rho\left(\left(a^{2}-V^{2}\right) \pi^{2}\left(y_{2}-y_{1}\right)^{2}+2 a^{2} x_{0}^{2}\right)}{2\left(1-\chi_{1}(t)\right)\left(y_{2}-y_{1}\right)\left(a^{2}-V^{2}\right) \pi^{2}} .
$$

Из неравенств $(2.25),(2.29)$ исключим функцию $\chi_{1}(t)$ :

$$
\begin{gathered}
\chi_{1}(t)>\frac{y_{1} V^{2} \rho\left(\left(a^{2}-V^{2}\right) \pi^{2}\left(y_{2}-y_{1}\right)^{2}+2 a^{2} x_{0}^{2}\right)}{\left(y_{2}-y_{1}\right)\left(2 K_{1}(t)\left(a^{2}-V^{2}\right) \pi^{2} y_{1}-V^{2} \rho\left(\left(a^{2}-V^{2}\right) \pi^{2} y_{1}^{2}+2 a^{2} x_{0}^{2}\right)\right)}, \\
\chi_{1}(t)<1-\frac{\left(y_{0}-y_{2}\right) V^{2} \rho\left(\left(a^{2}-V^{2}\right) \pi^{2}\left(y_{2}-y_{1}\right)^{2}+2 a^{2} x_{0}^{2}\right)}{\left(y_{2}-y_{1}\right)\left(2 K_{2}(t)\left(y_{0}-y_{2}\right)\left(a^{2}-V^{2}\right) \pi^{2}-V^{2} \rho\left(\left(a^{2}-V^{2}\right) \pi^{2}\left(y_{0}-y_{2}\right)^{2}+2 a^{2} x_{0}^{2}\right)\right)}
\end{gathered}
$$

Окончательно получим следующие условия:

$$
\begin{gathered}
\frac{y_{1} V^{2} \rho\left(\left(a^{2}-V^{2}\right) \pi^{2}\left(y_{2}-y_{1}\right)^{2}+2 a^{2} x_{0}^{2}\right)}{\left(y_{2}-y_{1}\right)\left(2 K_{1}(t)\left(a^{2}-V^{2}\right) \pi^{2} y_{1}-V^{2} \rho\left(\left(a^{2}-V^{2}\right) \pi^{2} y_{1}^{2}+2 a^{2} x_{0}^{2}\right)\right)} \in(0,1) ; \\
\frac{\left(y_{0}-y_{2}\right) V^{2} \rho\left(\left(a^{2}-V^{2}\right) \pi^{2}\left(y_{2}-y_{1}\right)^{2}+2 a^{2} x_{0}^{2}\right)}{\left(y_{2}-y_{1}\right)\left(2 K_{2}(t)\left(y_{0}-y_{2}\right)\left(a^{2}-V^{2}\right) \pi^{2}-V^{2} \rho\left(\left(a^{2}-V^{2}\right) \pi^{2}\left(y_{0}-y_{2}\right)^{2}+2 a^{2} x_{0}^{2}\right)\right)} \in(0,1)
\end{gathered}
$$




$$
\begin{gathered}
\frac{y_{1}}{\left(2 K_{1}(t) y_{1}\left(a^{2}-V^{2}\right) \pi^{2}-V^{2} \rho\left(\left(a^{2}-V^{2}\right) \pi^{2} y_{1}^{2}+2 a^{2} x_{0}^{2}\right)\right)}+ \\
+\frac{y_{0}-y_{2}}{\left(2 K_{2}(t)\left(y_{0}-y_{2}\right)\left(a^{2}-V^{2}\right) \pi^{2}-V^{2} \rho\left(\left(a^{2}-V^{2}\right) \pi^{2}\left(y_{0}-y_{2}\right)^{2}+2 a^{2} x_{0}^{2}\right)\right)}< \\
<\frac{\left(y_{2}-y_{1}\right)}{V^{2} \rho\left(\left(a^{2}-V^{2}\right) \pi^{2}\left(y_{2}-y_{1}\right)^{2}+2 a^{2} x_{0}^{2}\right)} .
\end{gathered}
$$

Так как при условиях (2.30), (2.31), (2.32) все квадратичные формы в (2.21) положительно определены, то из $(2.21)$ окончательно получим оценку $\Phi(t) \geqslant 0$, при этом $\dot{\Phi}(t) \leqslant 0$ согласно (2.14).

В монографии [2] доказана следующая лемма.

Лемма 1. Если можно построить такой функиионал $\Phi(t) \equiv \Phi\left(w_{1}, w_{2}, u_{1}, u_{2}, \varphi\right)$ полиномиального вида, где $w_{i}(x, t), u_{i}(x, t), i=1,2, \varphi(x, y, t)$-решение задачи $(2.1)-(2.10)$, что $\Phi(t) \geqslant 0$ $u \dot{\Phi}(t) \leqslant 0$, то функции $w_{i}(x, t), u_{i}(x, t), i=1,2, u$ (или) $\varphi(x, y, t)$ и (или) их производные, входлщие в положительно определенныи полином в выражении для $\Phi(t)$ или в положительно определенную ниюнюю оценку $\Phi_{1}(t)$ этого функиионала $\Phi(t)\left(\Phi(t) \geqslant \Phi_{1}(t) \geqslant 0\right)$, устойчивы по отношения к возмущениям начальных данных (2.9), (2.10).

Согласно лемме 1 имеет место следующая теорема.

Теорема 1. Пусть для любого момента времени $t>0$ выполняются условия (2.13), (2.30), (2.31), (2.32). Тогда решение $w_{i}(x, t), i=1,2, \varphi(x, y, t)$ задачи $(2.1)-(2.10)$ и производные $\varphi_{t}(x, y, t)$, $\varphi_{x}(x, y, t), \varphi_{y}(x, y, t), \dot{u}_{i}(x, t), w_{i}^{\prime}(x, t), \dot{w}_{i}(x, t), w_{i}^{\prime \prime}(x, t), i=1,2$, устойчивы по отношению к возмущениям начальных данных (2.9), (2.10).

\section{3. Динамическая устойчивость деформируемых элементов конструкций при сверх- звуковом режиме обтекания.}

Математическая модель. Пусть на плоскости $O x y$, в которой происходят совместные колебания потока идеального газа и упругой пластины, пластине соответствует положение $y=0$, $x \in[0, l]$. Скорость невозмущенного однородного потока параллельна оси $O x$ и равна $V(V>a$, где $a$-скорость звука в потоке, число Маха $M=V / a>1)$. На рис. 2 представлены примеры обтекания конструкций с упругим элементом сверхзвуковым потоком газа: а) двустороннее обтекание рассекателя с образованием ударной волны; б) одностороннее обтекание защитного экрана с образованием волны разрежения.

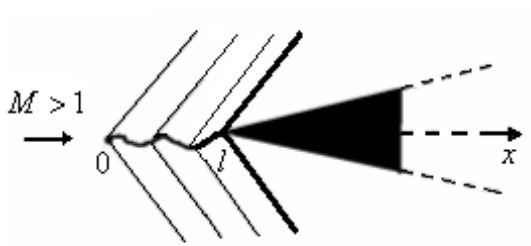

a)

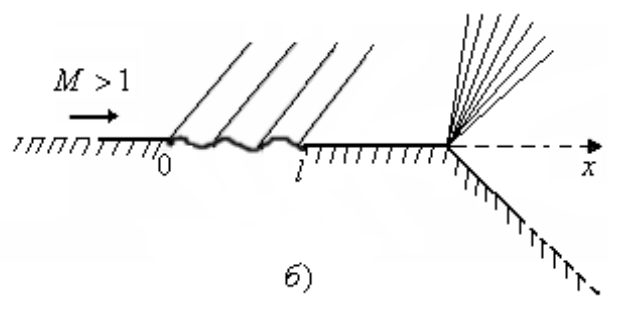

6)

Рис. 2. Примеры обтекания конструкций с упругим элементом сверхзвуковым потоком газа.

Рассмотрим нелинейную систему дифференциальных уравнений, описывающую продольнопоперечные колебания упругой пластины при обтекании ее сверхзвуковым потоком газа:

$$
\left\{\begin{array}{l}
-E F\left(u^{\prime}(x, t)+\frac{1}{2} w^{\prime 2}(x, t)\right)^{\prime}+m \ddot{u}(x, t)-\beta_{2} F \dot{u}^{\prime \prime}(x, t)=0, \\
-E F\left[w^{\prime}(x, t)\left(u^{\prime}(x, t)+\frac{1}{2} w^{\prime 2}(x, t)\right)\right]^{\prime}+D w^{\prime \prime \prime \prime}(x, t)+m \ddot{w}(x, t)+N(t) w^{\prime \prime}(x, t)+ \\
\quad+\beta_{2} I \dot{w}^{\prime \prime \prime \prime}(x, t)+\beta_{1} \dot{w}(x, t)+\beta_{0} w(x, t)+\alpha\left(\dot{w}(x, t)+V w^{\prime}(x, t)\right)=0, \quad x \in(0, l) .
\end{array}\right.
$$


Здесь $u(x, t)$ и $w(x, t)$ - деформации упругих элементов в направлении осей $O x$ и $O y$ соответственно; штрих обозначает производную по координате $x$, точка - производную по времени $t$; $h$ - толщина пластины; $E$ и $\rho$-модуль упругости и плотность материала пластины; $m=h \rho-$ погонная масса пластины; $N(t)$ - продольное усилие; $F=h /\left(1-\nu^{2}\right) ; D=E I$ - изгибная жесткость пластины; $I=h^{3} /\left(12\left(1-\nu^{2}\right)\right)$ - момент инерции; $\nu$-коэффициент Пуассона; $\beta_{2}$ и $\beta_{1}-$ коэффициенты внутреннего и внешнего демпфирования; $\beta_{0}$ - коэффициент жесткости слоя обжатия; $\alpha=\alpha_{0} \rho_{0} a_{0}=$ const $>0$, где $\rho_{0}$ и $a_{0}$-плотность газа и скорость звука в однородном невозмущенном потоке $\left(\alpha_{0}=1\right.$ при одностороннем обтекании, $\alpha_{0}=2$ при двустороннем обтекании); аэродинамическая нагрузка определяется выражением $\alpha\left(\dot{w}+V w^{\prime}\right)$, справедливым при достаточно больших скоростях сверхзвукового потока $V$, вывод которого представлен, например, в [8] и соответствует поршневой теории Ильюшина.

Исследуем устойчивость пластины в случае жесткого защемления обоих концов пластины:

$$
w(0, t)=w^{\prime}(0, t)=u(0, t)=w(l, t)=w^{\prime}(l, t)=u(l, t)=0 .
$$

Зададим начальные условия:

$$
w(x, 0)=f_{1}(x), \quad \dot{w}(x, 0)=f_{2}(x), \quad u(x, 0)=f_{3}(x), \quad \dot{u}(x, 0)=f_{4}(x),
$$

которые должны быть согласованы с граничными условиями (3.2). Таким образом, получена начально-краевая задача (3.1), (3.2), (3.3) для определения двух неизвестных функций $w(x, t), u(x, t)$. Сжимающая $(N>0)$ или растягивающая $(N<0)$ пластину сила $N$ может зависеть от времени.

Исследование устойчивости. Введем функционал

$$
\begin{aligned}
\Phi(t)= & \int_{0}^{l}\left\{E F\left(u^{\prime}(x, t)+\frac{1}{2} w^{\prime 2}(x, t)\right)^{2}+m\left(\dot{u}^{2}(x, t)+\dot{w}^{2}(x, t)\right)+\right. \\
& +D w^{\prime \prime 2}(x, t)-N(t) w^{\prime 2}(x, t)+\beta_{0} w^{2}(x, t)+4 m \theta u(x, t) \dot{u}(x, t)+2 m \theta \dot{w}(x, t) w(x, t)+ \\
& \left.+2 \beta_{2} F \theta u^{\prime 2}(x, t)+\beta_{1} \theta w^{2}(x, t)+\beta_{2} I \theta w^{\prime \prime 2}(x, t)+\alpha \theta w^{2}(x, t)\right\} d x,
\end{aligned}
$$

где $\theta>0$ - некоторый постоянный положительный параметр.

Проведем оценки для функционала с учетом граничных условий (3.2). Используя неравенство Рэлея, получим:

$$
\begin{array}{ll}
\int_{0}^{l} w^{\prime \prime 2}(x, t) d x \geqslant \lambda_{1} \int_{0}^{l} w^{\prime 2}(x, t) d x, & \int_{0}^{l} u^{\prime 2}(x, t) d x \geqslant \eta_{1} \int_{0}^{l} u^{2}(x, t) d x, \\
\int_{0}^{l} w^{\prime 2}(x, t) d x \geqslant \vartheta_{1} \int_{0}^{l} w^{2}(x, t) d x, & \int_{0}^{l} \dot{w}^{\prime \prime 2}(x, t) d x \geqslant \mu_{1} \int_{0}^{l} \dot{w}^{2}(x, t) d x,
\end{array}
$$

где $\lambda_{1}, \mu_{1}, \vartheta_{1}$ - наименьшие собственные значения краевых задач

$$
\phi^{\prime \prime \prime \prime}=-\lambda \phi^{\prime \prime}, \quad \phi^{\prime \prime \prime \prime}=\mu \phi, \quad \phi^{\prime \prime}=-\vartheta \phi
$$

с краевыми условиями (3.2) для функции $w(x, t), \eta_{1}$ - наименьшее собственное значение краевой задачи $\phi^{\prime \prime}=-\eta \phi$ с краевыми условиями (3.2) для функции $u(x, t)$.

Учитывая, что первое слагаемое под знаком интеграла в функционале (3.4) неотрицательно, и применяя первое неравенство (3.5), получим: 


$$
\begin{aligned}
& \Phi(t) \geqslant \int_{0}^{l}\left\{m\left(\dot{u}^{2}(x, t)+\dot{w}^{2}(x, t)\right)+\right. \\
& \quad+\left(\lambda_{1}\left(D+\beta_{2} I \theta\right)-N(t)\right) w^{\prime 2}(x, t)-\left(\beta_{0}+\beta_{1} \theta+\alpha \theta\right) w^{2}(x, t)+ \\
& \left.\quad+4 m \theta u(x, t) \dot{u}(x, t)+2 m \theta \dot{w}(x, t) w(x, t)+2 \beta_{2} F \theta u^{\prime 2}(x, t)\right\} d x .
\end{aligned}
$$

Пусть выполняются следующие условия:

$$
m>0, \quad D>0, \quad \beta_{0} \geqslant 0, \quad \beta_{1} \geqslant 0, \quad \beta_{2} \geqslant 0, \quad D \lambda_{1}-N(t)>0 .
$$

Тогда, используя второе и третье неравенства (3.5), получим следующую оценку функционала:

$$
\begin{aligned}
\Phi(t) \geqslant \int_{0}^{l}\left\{m \dot{u}^{2}(x, t)+\right. & 4 m \theta u(x, t) \dot{u}(x, t)+ \\
& +2 \beta_{2} F \theta \eta_{1} u^{2}(x, t)+m \dot{w}^{2}(x, t)+2 m \theta w(x, t) \dot{w}(x, t)+ \\
& \left.+\left[\beta_{0}+\beta_{1} \theta+\alpha \theta+\vartheta_{1}\left(\lambda_{1}\left(D+\beta_{2} I \theta\right)-N(t)\right)\right] w^{2}(x, t)\right\} d x .
\end{aligned}
$$

Получили две квадратичные формы относительно $u(x, t), \dot{u}(x, t)$ и $w(x, t), \dot{w}(x, t)$. Согласно критерию Сильвестра квадратичные формы будут положительно определенными, если выполняются условия

$$
\beta_{2} F \eta_{1}-2 m \theta>0, \quad \beta_{0}+\beta_{1} \theta+\alpha \theta+\vartheta_{1}\left[\lambda_{1}\left(D+\beta_{2} I \theta\right)-N(t)\right]>m \theta^{2} .
$$

Следовательно, получено неравенство $\Phi(t) \geqslant 0$.

Начальное значение функционала примет вид

$$
\begin{aligned}
\Phi(0)= & \int_{0}^{l}\left\{E F\left(u^{\prime}(x, 0)+\frac{1}{2} w^{\prime 2}(x, 0)\right)^{2}+m\left(\dot{u}^{2}(x, 0)+\dot{w}^{2}(x, 0)\right)+\right. \\
+\left(D+\beta_{2} I \theta\right) w^{\prime \prime 2}(x, 0)-N(0) w^{\prime 2}(x, 0)+ & \left(\beta_{0}+\beta_{1} \theta+\alpha \theta\right) w^{2}(x, 0)+4 m \theta u(x, 0) \dot{u}(x, 0)+ \\
& \left.+2 m \theta w(x, 0) \dot{w}(x, 0)+2 \beta_{2} F \theta u^{\prime 2}(x, 0)\right\} d x .
\end{aligned}
$$

Найдем производную от функционала (3.4) по переменной $t$ :

$$
\begin{gathered}
\dot{\Phi}(t)=\int_{0}^{l}\left\{2 E F\left(u^{\prime}(x, t)+\frac{1}{2} w^{\prime 2}(x, t)\right)\left(\dot{u}^{\prime}(x, t)+w^{\prime}(x, t) \dot{w}^{\prime}(x, t)\right)+2 m \dot{u}(x, t) \ddot{u}(x, t)+\right. \\
+2 m \dot{w}(x, t) \ddot{w}(x, t)+2 D w^{\prime \prime}(x, t) \dot{w}^{\prime \prime}(x, t)-\dot{N}(t) w^{\prime 2}(x, t)- \\
-2 N(t) w^{\prime}(x, t) \dot{w}^{\prime}(x, t)+2 \beta_{0} w(x, t) \dot{w}(x, t)+4 m \theta \dot{u}^{2}(x, t)+4 m \theta u(x, t) \ddot{u}(x, t)+ \\
+2 m \theta \dot{w}^{2}(x, t)+2 m \theta w(x, t) \ddot{w}(x, t)+4 \beta_{2} F \theta u^{\prime}(x, t) \dot{u}^{\prime}(x, t)+ \\
\left.+2 \beta_{1} \theta w(x, t) \dot{w}(x, t)+2 \beta_{2} I \theta w^{\prime \prime}(x, t) \dot{w}^{\prime \prime}(x, t)+2 \alpha \theta w(x, t) \dot{w}(x, t)\right\} d x .
\end{gathered}
$$


Для функций $u(x, t), w(x, t)$, удовлетворяющих системе (3.1) и условиям $(3.2)$, из (3.12) получим равенство

$$
\begin{aligned}
\dot{\Phi}(t)= & -2 \int_{0}^{l}\left\{\beta_{2} F \dot{u}^{\prime 2}(x, t)-2 m \theta \dot{u}^{2}(x, t)\right. \\
+\beta_{2} I \dot{w}^{\prime 2}(x, t)+\alpha V \dot{w}(x, t) w^{\prime}(x, t)+ & 2 \theta E F\left(u^{\prime}(x, t)+\frac{1}{2} w^{\prime 2}(x, t)\right)^{2}+\theta D w^{\prime \prime 2}(x, t)+ \\
& +\left(\frac{1}{2} \dot{N}(t)-\theta N(t)\right) \dot{w}^{2}(x, t)+ \\
&
\end{aligned}
$$

Используя оценки (3.5), будем иметь:

$$
\begin{aligned}
\dot{\Phi}(t) \leqslant-2 \int_{0}^{l}\left\{\left(\beta_{2} F \eta_{1}-2 m \theta\right)\right. & \dot{u}^{2}(x, t)+\left(\beta_{2} I \mu_{1}+\beta_{1}+\alpha-m \theta\right) \dot{w}^{2}(x, t)+ \\
& \left.+\alpha V \dot{w}(x, t) w^{\prime}(x, t)+\left(\theta D \lambda_{1}+\frac{1}{2} \dot{N}(t)-\theta N(t)\right) w^{\prime 2}(x, t)\right\} d x .
\end{aligned}
$$

Получили две квадратичные формы относительно $\dot{u}(x, t)$ и $\dot{w}(x, t), w^{\prime}(x, t)$. Квадратичные формы с учетом (3.10) будут положительно полуопределенными, если выполняются условия

$$
\begin{gathered}
\beta_{2} I \mu_{1}+\beta_{1}+\alpha-m \theta>0, \\
4\left(\beta_{2} I \mu_{1}+\beta_{1}+\alpha-m \theta\right)\left(\theta D \lambda_{1}+\frac{1}{2} \dot{N}(t)-\theta N(t)\right)-\alpha^{2} V^{2} \geqslant 0 .
\end{gathered}
$$

Следовательно, при выполнении первого условия (3.10) и условий (3.15), (3.16) из (3.14) получим оценку

$$
\dot{\Phi}(t) \leqslant 0 .
$$

Исключая из системы (3.8), (3.10), (3.15), (3.16) параметр $\theta$, окончательно получим условия устойчивости в следующем виде:

$$
\begin{gathered}
m>0, \quad D>0, \quad \beta_{0} \geqslant 0, \quad \beta_{1} \geqslant 0, \quad \beta_{2} \geqslant 0, \quad D_{1}(t)>0, \\
2 \alpha_{1} D_{1}(t)+m \dot{N}(t)>2 \alpha V \sqrt{m D_{1}(t)}, \\
\max \left\{0, \sup _{t} \frac{2 \alpha_{1} D_{1}(t)-m \dot{N}(t)-\sqrt{\left(2 \alpha_{1} D_{1}(t)+m \dot{N}(t)\right)^{2}-4 m \alpha^{2} V^{2} D_{1}(t)}}{4 m D_{1}(t)}\right\}< \\
<\min \left\{\frac{\alpha_{1}}{m}, \frac{\beta_{2} F \eta_{1}}{2 m}, \inf _{t} \frac{\alpha_{2}+\sqrt{\alpha_{2}^{2}+4 m\left(\vartheta_{1} D_{1}(t)+\beta_{0}\right)}}{2 m},\right. \\
\left.\inf _{t} \frac{2 \alpha_{1} D_{1}(t)-m \dot{N}(t)+\sqrt{\left(2 \alpha_{1} D_{1}(t)+m \dot{N}(t)\right)^{2}-4 m \alpha^{2} V^{2} D_{1}(t)}}{4 m D_{1}(t)}\right\}
\end{gathered}
$$

где $\alpha_{1}=\beta_{2} I \mu_{1}+\beta_{1}+\alpha, \alpha_{2}=\beta_{2} I \vartheta_{1} \lambda_{1}+\beta_{1}+\alpha, D_{1}(t)=D \lambda_{1}-N(t)$. Условия (3.18) явно содержат параметры механической системы.

Из леммы 1 вытекает следующее утверждение. 
Лемма 2. Если можно построить такой функиионал $\Phi(t) \equiv \Phi(w, u)$ полиномиального вида, где $w(x, t), u(x, t)$ - решение задачи $(3.1)-(3.3)$, что $\Phi(t) \geqslant 0, \dot{\Phi}(t) \leqslant 0$, то функиии $w(x, t), u(x, t)$ $u$ (или) их производные, входящие в положительно определенный полином в выражении для $\Phi(t)$ или в положительно определенную нижнюю оценку $\Phi_{1}(t)$ этого функционала $\Phi(t)(\Phi(t) \geqslant$ $\left.\Phi_{1}(t) \geqslant 0\right)$, устойчивы по отношению $\kappa$ возмущениям начальных данных (3.3).

Из леммы 2 получаем следующую теорему.

Теорема 2. Пусть для любого момента времени $t>0$ выполняются условия (3.18). Тогда решение $w(x, t), u(x, t)$ задачи (3.1)-(3.3) и производные $\dot{u}(x, t), \dot{w}(x, t)$ устойчивы по отношению $\kappa$ возмущениям начальных данных (3.3).

4. Заключение. На основе предложенных математических моделей продольно-поперечных колебаний упругих элементов конструкций в виде пластины-полосы при обтекании их дозвуковым и сверхзвуковым потоком идеального газа проведены исследования динамической устойчивости деформируемых элементов вибрационных устройств при дозвуковом режиме обтекания и динамической устойчивости упругого защитного экрана при сверхзвуковом обтекании. Модели описываются нелинейными системами дифференциальных уравнений с частными производными. С помощью построенных функционалов получены достаточные условия устойчивости решений этих систем уравнений. Полученные условия устойчивости накладывают ограничения на погонные массы и изгибные жесткости элементов, сжимающие (растягивающие) элементы усилия, скорость невозмущенного однородного потока, коэффициенты внутреннего и внешнего демпфирования и другие параметры механических систем. Эти условия явно содержат основные параметры механических систем, и в таком виде они наиболее приспособлены для решения задач оптимизации, автоматического управления, автоматизированного проектирования.

\section{СПИСОК ЛИТЕРАТУРЫ}

1. Алгазин С. Д., Кийко И. А. Флаттер пластин и оболочек. - М.: Наука, 2006.

2. Анкилов А. В., Велъмисов П. А. Функционалы Ляпунова в некоторых задачах динамической устойчивости аэроупругих конструкций. - Ульяновск: УлГТУ, 2015.

3. Анкилов А. В., Вельмисов П. А. Математическое моделирование в задачах динамической устойчивости деформируемых элементов конструкций при аэрогидродинамическом воздействии. - Ульяновск: УлГТУ, 2013.

4. Анкилов A. B., Велъмисов П. А. Динамика и устойчивость упругих пластин при аэрогидродинамическом воздействии. - Ульяновск: УлГТУ, 2009.

5. Анкилов А. В., Вельмисов П. А., Горбоконенко В. Д., Покладова Ю. В. Математическое моделирование механической системы «трубопровод-датчик давления». - Ульяновск: УлГТУ, 2008.

6. Анкилов А. В., Вельмисов П. А. Исследование динамики и устойчивости упругого элемента конструкции при сверхзвуковом обтекании// Вестн. Саратов. гос. техн. ун-та. - 2011. - 3 (57), № 1. - С. $59-67$.

7. Анкилов А. В., Вельмисов П. А. Исследование устойчивости вязкоупругого элемента конструкции при сверхзвуковом обтекании // Ж. Средневолж. мат. об-ва. - 2016. - 18, № 3. - С. 80-90.

8. Вельмисов П. А., Судаков В. А., Анкилов А. В. Численный эксперимент в задаче о динамике защитного экрана при сверхзвуковом обтекании потоком газа// Вестн. Ульяновск. гос. техн. ун-та. - 2013. - 3. - C. 38-44.

9. Гувернюк С. В., Зубков А. Ф., Симоненко М. М. Экспериментальное исследование сверхзвукового обтекания осесимметричной кольцевой каверны// Инж.-физ. ж. - 2016. - 89, № 3. - С. 670-679.

10. Кийко И. А., Показеев В. В. К постановке задачи о колебаниях и устойчивости полосы в сверхзвуковом потоке газа// Мех. жидк. газа. - 2009. - 1. - С. 159-166.

11. Коллати, Л. Задачи на собственные значения. - М.: Наука, 1968.

12. Могилевич Л. И., Попов В. С., Попова А. А., Христоборова А. В. Математическое моделирование динамики взаимодействия сильновязкой жидкости со стенками канала, установленного на упругом основании // Динам. сист. мех. машин. - 2016. - 3, № 1. - С. 350-354.

13. Ряховский А. И., Шмидт А. А. Численное моделирование МГД управления сверхзвуковым потоком в среде OpenFOAM// Тр. ИСП РАН. - 2016. - 28, № 1. - С. 197-206.

14. Ankilov A. V., Vel'misov P. A. Stability of solutions to an aerohydroelasticity problem// J. Math. Sci. 2016. - 219, № 1. - P. 14-26. 
15. Askari E., Jeong K. H., Amabili M. Hydroelastic vibration of circular plates immersed in a liquid-filled container with free surface// J. Sound Vibration. — 2013. — 332, № 12. — P. 3064-3085.

16. Aulisa E., Ibragimov A., Kaya-Cekin E. Y. Fluid structure interaction problem with changing thickness beam and slightly compressible fluid// Discr. Contin. Dyn. Syst. Ser. S. - 2014. — 7, № 6. - P. 1133-1148.

17. Baghdasaryan G. Y., Mikilyan M. A., Saghoyan R. O. Influence of supersonic gas flow on the amplitude of non-linear oscillations of rectangular plates// Mechanics. Proc. Natl. Acad. Sci. Armenia. - 2016. - 69, № 4. - P. 20-40.

18. Brehm C., Housman J., Kiris C. Noise generation mechanisms for a supersonic jet impinging on an inclined plate// J. Fluid Mech. — 2016. - 797. - P. 802-850.

19. Faal R. T., Derakhshan D. Flow-Induced Vibration of Pipeline on Elastic Support// Proc. Eng. — 2011. - 14. - P. 2986-2993.

20. Filippi A., Skews B. Supersonic flow fields resulting from axisymmetric internal surface curvature// J. Fluid Mech. - 2017. - 831. - P. 271-288.

21. Gatica G. N., Heuer N., Meddahi S. Coupling of mixed finite element and stabilized boundary element methods for a fluid-solid interaction problem in 3D// Numer. Meth. Partial Differ. Equations. — 2014. 30, № 4. - P. 1211-1233.

22. Gounko Y. P. Patterns of steady axisymmetric supersonic compression flows with a Mach disk// Shock Waves. - 2017. - 27, № 3. - P. 495-506.

23. Kounadis $A$. $N$. Flutter instability and other singularity phenomena in symmetric systems via combination of mass distribution and weak damping// Int. J. Nonlin. Mech. — 2007. — 42, № 1. - P. 24-35.

24. Kontzialis K., Moditis K., Paidoussis M. P. Transient simulations of the fluid-structure interaction response of a partially confined pipe under axial flows in opposite directions// J. Pressure Vessel Tech. — 2017. 139, № 3. - P. 1-8.

25. Moditis K., Paidoussis M., Ratigan J. Dynamics of a partially confined, discharging, cantilever pipe with reverse external flow// J. Fluids Struct. - 2016. - 63. - P. 120-139.

26. Vedeneev $V$. V. Effect of damping on flutter of simply supported and clamped panels at low supersonic speeds// J. Fluids Struct. — 2013. - 40. - P. 366-372.

27. Willems S., Gulhan A., Esser B. Shock induced fluid-structure interaction on a flexible wall in supersonic turbulent flow// Progr. Flight Phys. - 2013. - 5. - P. 285-308.

Вельмисов Петр Александрович

Ульяновский государственный технический университет

E-mail: velmisov@ulstu.ru

Анкилов Андрей Владимирович

Ульяновский государственный технический университет

E-mail: ankil@ulstu.ru

Покладова Юлия Валерьевна

Ульяновский государственный технический университет

E-mail: pokladovau@inbox.ru 